\title{
Regional Brain Glucose Metabolism and Its Prognostic Value in Pretreatment Extranodal Natural Killer/T-Cell Lymphoma Patients
}

\author{
Ziwei Zhou* \\ Zhe Guo* \\ Qingqiao $\mathrm{Hu}$ \\ Wei Ding \\ Chongyang Ding \\ Lijun Tang (D)
}

Department of Nuclear Medicine, The First Affiliated Hospital of Nanjing Medical University, Nanjing, 210029 , Jiangsu, People's Republic of China

*These authors contributed equally to this work
Correspondence: Lijun Tang;

Chongyang Ding

Department of Nuclear Medicine, The First Affiliated Hospital of Nanjing Medical University, No. 300, Guangzhou Road,

Nanjing, 210029, People's Republic of China

Tel +|475|830090; +|595|6|4327

Email tanglijun@njmu.edu.cn;

chongyangding@163.com
Objective: To explore regional brain glucose metabolic abnormalities of pretreatment stage I/II extranodal natural killer/T-cell lymphoma (ENKTL) patients using positron emission tomography with 2-deoxy-2-[fluorine-18]fluoro-D-glucose integrated with computed tomography $\left({ }^{18} \mathrm{~F}-\mathrm{FDG} \mathrm{PET} / \mathrm{CT}\right)$ and assess its prognostic value.

Methods: Sixty pretreatment stage I/II ENKTL patients were enrolled in this retrospective study and divided into survival $(n=45)$ and death $(n=15)$ groups according to their status at the end of follow-up. A control group consisted of 60 healthy subjects. Regional cerebral glucose metabolism was evaluated on a voxel-by-voxel basis using statistical parametric mapping (SPM8) under a certain significance level $(\mathrm{P}<0.001)$ and voxel threshold $(\mathrm{K}=100$ voxels).

Results: Decreased metabolism was noted in patients, involving the bilateral prefrontal and orbitofrontal cortex, partial parietal and occipital cortex, cingulate gyrus and cerebellum; the sensorimotor cortex was largely spared. Increased metabolism was observed in the bilateral putamen, amygdala, and parahippocampal gyrus. Compared with the survival group, the death group had higher metabolism in the bilateral amygdala, putamen, left thalamus, uncus, and parahippocampal gyrus. Only B symptoms were associated with the increased metabolism of basal ganglia and thalamus (BGT). Patients with high metabolic tumor volume, total lesion glycolysis (TLG) and BGT metabolism had a poor prognosis. TLG and maximum standardized uptake value (SUVmax) LBGT/SUVmaxRight cerebellum were associated with Eastern Cooperative Oncology Group (ECOG) and prognostic index of natural killer lymphoma and Epstein-Barr virus-DNA (PINKE) scores. In multivariate analysis, only ECOG was an independent prognostic factor of both progression-free survival (PFS) and overall survival (OS). PINKE was an independent prognostic factor of OS.

Conclusion: Pretreatment stage I/II ENKTL patients exhibited abnormal regional cerebral glucose metabolism. Higher pretreatment glucose metabolism in BGT could predict a relatively poor prognosis but did not surpass the predictive values of ECOG and PINKE in stage I/II ENKTL patients.

Keywords: extranodal natural killer/T- cell lymphoma, regional cerebral glucose metabolism, ${ }^{18} \mathrm{~F}$-FDG PET/CT, statistical parametric mapping, prognostic value

\section{Introduction}

Abnormal brain glucose metabolism has parenthetically been observed and increasingly reported in patients with benign and malignant diseases even when the brain is spared. ${ }^{1-4}$ Other studies have reported that there is a statistically significant negative correlation between the standard uptake value (SUV) in the brain and total lesion glycolysis (TLG) in patients with malignant diseases. Such decreased brain uptake has 
been attributed to competition between the brain and hypermetabolic tumor tissues. ${ }^{5,6}$ However, abnormal regional brain metabolism including increased metabolism was found in pretreatment early stage (I/II) extranodal natural killer/T-cell lymphoma (ENKTL) patients with limited lesions, as well as low TLG in our daily work (Figure 1), which could not be explained by the above mechanism.

Risk restratification of ENKTL patients is very important for clinical management. Some stage I/II ENKTL patients have a poor prognosis, although the overall prognosis was thought to be relatively satisfactory. To date, there is no recognized prognostic indicator or model that provides specific risk stratification for ENKTL patients, let alone stage I/II ENKTL patients. The most common approaches include the use of the International Prognostic Index (IPI), ${ }^{7}$ the Korean Prognostic Index
$(\mathrm{KPI}){ }^{8}$ and the prognostic index of natural killer lymphoma and Epstein-Barr virus-DNA (PINKE). ${ }^{9}$ Additionally, maximum SUV (SUVmax), metabolic tumor volume (MTV), and TLG on baseline PET/CT ${ }^{10,11}$ and the Deauville 5-point scale (DS) on interim and end-of -treatment PET/CT may predict survival. ${ }^{12}$ However, all of these prognostic indicators have disadvantages. According to IPI and KPI scores, most patients categorized as low risk had poor clinical outcomes. They are unable to identify patients with more aggressive disease within the lowrisk category. ${ }^{13}$ Two studies concluded that the predictive values of SUVmax, MTV, and TLG before treatment were quite limited, mainly due to the unique features of ENKTL, no standard calculation method, no recognized optimal cut-off value, and other factors. ${ }^{10,12}$ Interim PET/ CT evaluation interpreted by DS is considered to have
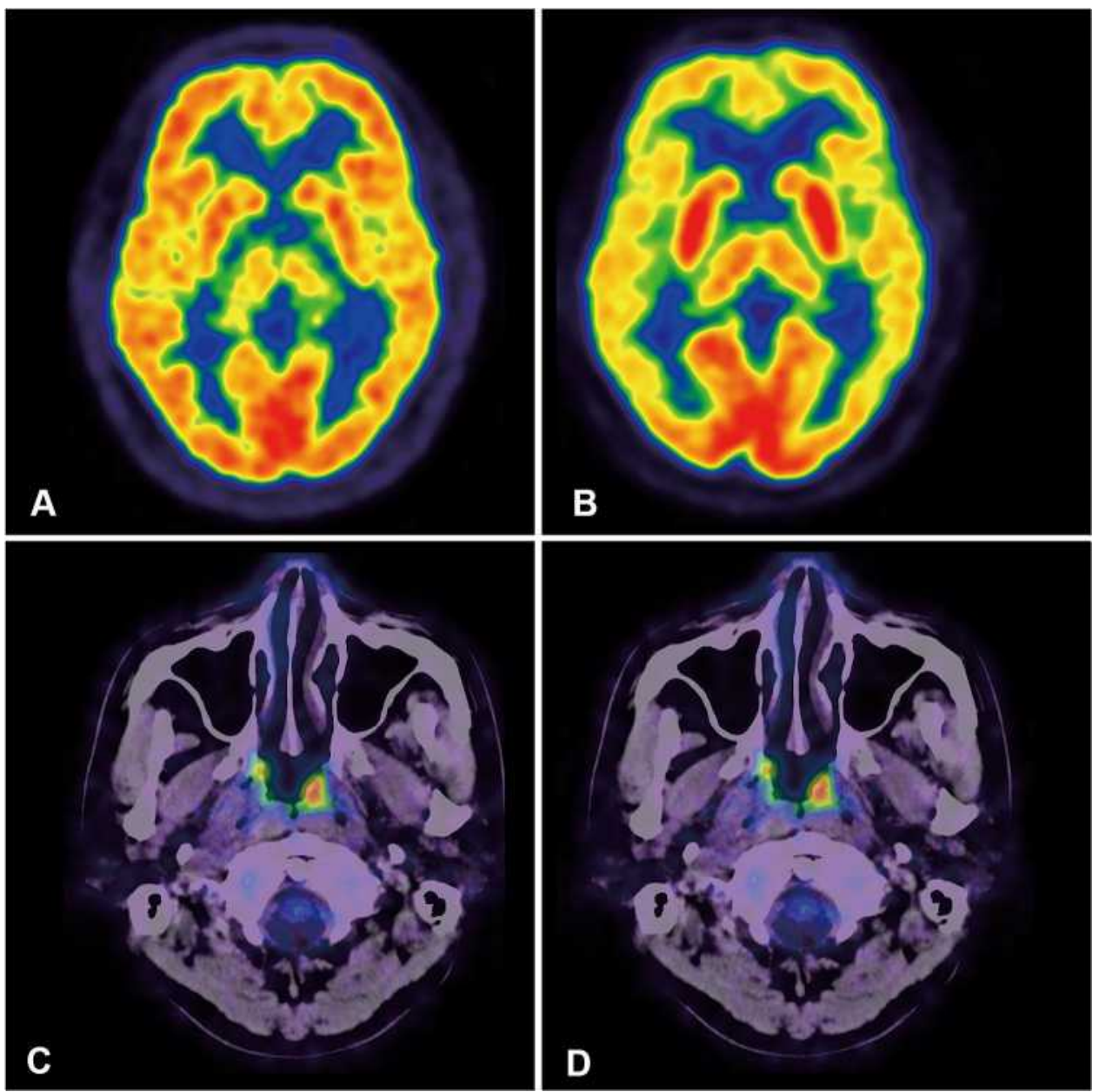

Figure I Cerebral ${ }^{18}$ F-FDG Metabolism of a healthy control (A) and a patient (B-D). A 53-year-old male underwent ${ }^{18} \mathrm{~F}$-FDG PET/CT (A) during a health check, which showed relatively symmetric cerebral ${ }^{18} \mathrm{~F}$-FDG metabolism. No obvious abnormal metabolism was observed. ${ }^{18} \mathrm{~F}$-FDG metabolism in the basal ganglia was similar to that in the cortex. A 58-year-old male was diagnosed with stage II nasal type ENKTL, and pretreatment ${ }^{18} \mathrm{~F}$-FDG PET/CT revealed soft tissue in the left nasal cavity (C) and swollen lymph nodes in the right neck (D) with increased ${ }^{18} \mathrm{~F}$-FDG uptake. His pretreatment TLG was 36.9. Brain ${ }^{18} \mathrm{~F}$-FDG PET images demonstrated prominent hypometabolism in the bilateral frontal, parietal, and temporal cortices, with relative sparing of the occipital cortex. The metabolism of the bilateral basal ganglia was prominently higher than the cortex (B). The patient had been disease free for 30 months at the end of follow-up. 
a certain prognostic value ${ }^{12}$ but cannot be used before treatment. It is therefore essential to develop additional new approaches to restratify and reclassify stage I/II ENKTL patients. Different post-treatment cerebral glucose metabolism patterns were observed in patients with Hodgkin's lymphoma with different therapeutic response. $^{5}$ It is unclear whether different pretreatment brain glucose metabolism patterns exist in stage I/II ENKTL patients with different prognoses and whether pretreatment brain glucose metabolism patterns can be used as new approaches for risk restratification of this population.

The main purposes of the present study were to identify brain regions with abnormal metabolism in pretreatment, early stage I/II ENKTL patients; investigate underlying mechanisms that may influence regional brain metabolism; and determine the potential value of pretreatment regional brain glucose metabolism and baseline PET/ CT parameters in predicting prognoses for stage I/II ENKTL patients.

\section{Materials and Methods}

\section{Subjects}

Positron emission tomography with 2-deoxy-2-[fluorine-18] fluoro-D-glucose integrated with computed tomography $\left({ }^{18} \mathrm{~F}-\mathrm{FDG} \mathrm{PET} / \mathrm{CT}\right)$ scanning was performed in accordance with the Declaration of Helsinki. Use of data for retrospective analysis was approved by the Ethical Committee of The First Affiliated Hospital of Nanjing Medical University, and the need for informed consent was waived because this was a retrospective study and the data used were obtained from previous clinical practice. We ensure the confidentiality of all data was maintained at all times. Inclusion criteria were as follows: newly diagnosed and histologically confirmed stage I/II ENKTL and the use of pegaspargase, gemcitabine, oxaliplatin, and dexamethasone (PGOD) chemotherapy combined with radiotherapy as the first-line treatment. The exclusion criteria were as follows: (a) primary cerebral lymphoma or apparent focal brain lesions on MRI and CT images, (b) secondary hemophagocytic syndrome, (c) history of neurological or psychiatric disease or symptoms including insomnia, (d) history of alcoholism or psychotropic drugs usage, (e) history of previously treated other malignancy, (f) blood sugar level $>120 \mathrm{mg} / \mathrm{dl}$ to avoid effects of hyperglycemia on the brain, (g) history of diabetes mellitus, (h) apparent mis-registration between the CT and FDG-PET images. Finally, we enrolled 60 patients (average: 49.7 years old, range, 15-87) with newly diagnosed and histologically proven stage I/II nasal type ENKTL who had undergone ${ }^{18}$ F-FDG PET/CT for pretreatment staging between September 2010 and May 2018. Our investigation also included a control group (CG) with 60 subjects (average: 48.9 years old, range: $13-89$ ) without malignant tumors or other severe diseases (especially metabolic conditions) who underwent health checks with ${ }^{18}$ F-FDG-PET/CT in the same period. The general exclusion criteria were the same as for the patients.

\section{${ }^{18}$ F-FDG PET/CT Image Acquisition}

Subjects were required to fast $>6 \mathrm{~h}$ to reduce serum glucose $<7.0 \mathrm{mmol} / \mathrm{L}$. The participants were asked to rest quietly for $30 \mathrm{~min}$ prior to being injected with ${ }^{18} \mathrm{~F}$-FDG 3.70-5.55 MBq/kg and then rest for $1 \mathrm{~h}$. The whole-body and brain PET/CT scan were performed 60 minutes after ${ }^{18}$ F-FDG injection. During the examination, subjects were required to lie still in a dark quiet room during PET data acquisition, but they were free to close and open their eyes. The brain PET scan (120 s/bed position) included a low-amperage CT scan (120 KV and $380 \mathrm{~mA}$ ) for attenuation correction. PET images were acquired using a Siemens Biograph 16 PET/CT HR scanner in 3-dimensional mode over a period of $10 \mathrm{~min}$ after the whole-body PET scan. The data were reconstructed by iterative reconstruction (matrix, $256 \times 256$; thickness, $5 \mathrm{~mm}$ ). The tomography of the transverse, sagittal, and coronal planes, and fusion images were obtained after iterative reconstruction.

\section{PET/CT Image Analysis}

Regions of interest (ROIs) were drawn manually along the lesion edges. A threshold setting of $40 \%$ of the SUVmax was applied. The quantitative parameters were automatically calculated including SUVmax, metabolic tumor volume (MTV), and TLG (MTV $\times$ SUVmean) of ROIs. Brain PET/CT data preprocessing and statistical analysis were conducted using statistical parametric mapping 8 (SPM) software implemented in MATLAB 7.0. Standardization and smoothing were executed for the data preprocessing, smoothed using Gaussian kernel of $4 * 4 * 4 \mathrm{~mm}$ (full width at half maximum), followed by statistical analysis including parameter setting and parameter estimation staging to test the null hypothesis. Only clusters containing $>100$ contiguous voxels were accepted as significant. To explain differences in brain binding affinity among subjects, a grand mean scaled value of 50 was selected as a unified standard. On the basis of the 
SPM analysis and in accordance with the $\mathrm{p}<0.001$ threshold level and the voxel threshold $(\mathrm{K}=100)$, the different metabolic regions were projected onto a $3 \mathrm{D}$ image and Talairach coordinates. The PET findings were superimposed on an MRI template to ensure accurate identification of the affected structures. According to the SPM results, ROIs were placed over the basal ganglia and thalamus (BGT), regions around uncus, and cerebellum on each side in a blinded manner. The SUVmax values of ROIs were obtained automatically. The SUVmax ratios of BGT and the region around the uncus to the contralateral cerebellum were calculated on each side as semiquantitative parameters for statistical analysis.

\section{Statistical Analysis}

Non-normal distribution data are described as median (range). Group differences between plasma glucose, injected dose/weight, and PET/CT quantitative parameters in the patients/controls and survival/death subgroups were analyzed by Mann-Whitney $U$-tests. The optimal cut-off values for the PET/CT quantitative and semiquantitative parameters were obtained by use of the ROC analysis for overall survival (OS). Differences between subgroups according to clinical characteristics were analyzed by $X^{2}$ or Fisher exact tests. Multiple linear regression analysis was used to identify factors that may affect brain metabolism. Regional cerebral glucose metabolic parameters were dichotomized into low and high values according to the cut-off values/medians in each of these groups. KaplanMeier survival curves were used to estimate progression free survival (PFS) and OS. Comparisons of OS and PFS between subgroups were checked using Log rank tests. Then, Cox proportional hazards regression model were employed in multivariate analyses using factors with $\mathrm{P}<0.1$ on univariate analysis. Statistical analysis was carried out with software package SPSS 26.0. $\mathrm{P}<0.05$ (twosided) were considered statistically significant.

\section{Results}

\section{Clinical Characteristics}

The demographics and clinical characteristics of patients and controls are listed in Table 1. There were no significant statistical differences in clinical characteristics between ENKTL patients and CG subjects. All patients were classified as stage I (28 patients) or stage II (32 patients) according to the revised Ann Arbor classification proposed by Cotswold. ${ }^{14}$ All patients received PGOD and
Table I Characteristics of Control Subjects and Patients

\begin{tabular}{|c|c|c|c|}
\hline Variables & $\begin{array}{l}\text { Patients } \\
(n=60)\end{array}$ & $\begin{array}{l}\text { Controls } \\
(n=60)\end{array}$ & $P$ value \\
\hline Age $(y$, mean $\pm S D)$ & $49.7 \pm 14.6$ & $48.9 \pm 14.7$ & $0.775^{\mathrm{a}}$ \\
\hline $\operatorname{Sex}(M / F)$ & $42 / 18$ & $37 / 23$ & $0.336^{\mathrm{b}}$ \\
\hline Plasma glucose, $\mathrm{mmol} / \mathrm{L}$ & $5.8 \pm 0.9$ & $5.7 \pm 1.1$ & $0.684^{\mathrm{a}}$ \\
\hline $\begin{array}{l}\text { Injected dose/weight }(\mathrm{MBq} / \\
\mathrm{kg})^{\mathrm{c}} \\
\text { Median } \\
\text { Interquartile range }\end{array}$ & $\begin{array}{l}0.110 \\
0.100-0.128\end{array}$ & $\begin{array}{l}0.120 \\
0.110-0.130\end{array}$ & $0.061^{c}$ \\
\hline Stage (I/II) & $28 / 32$ & - & \\
\hline B symptoms (No/Yes) & $36 / 24$ & - & \\
\hline ECOG PS (0/I-2) & $45 / 15$ & - & \\
\hline LDH $(\leq \mathrm{ULN} />\mathrm{ULN})$ & $48 / 12$ & & \\
\hline EBVDNA ( $\leq$ ULN/>ULN) & $47 / 13$ & & \\
\hline$\beta 2-M G(\leq U L N />U L N)$ & $35 / 25$ & & \\
\hline aalPI/IPI (0/I-2) & $38 / 22$ & & \\
\hline PINKE (0/I-2) & $35 / 25$ & & \\
\hline $\begin{array}{l}\text { SUVmax } \\
\text { Median } \\
\text { Interquartile range }\end{array}$ & $\begin{array}{l}12.2 \\
8.5-16.1\end{array}$ & $\begin{array}{l}- \\
- \\
-\end{array}$ & \\
\hline $\begin{array}{l}\text { MTV }\left(\mathrm{cm}^{3}\right) \\
\quad \text { Median } \\
\text { Interquartile range }\end{array}$ & $\begin{array}{l}12.2 \\
7.1-20.9\end{array}$ & $\begin{array}{l}- \\
- \\
-\end{array}$ & \\
\hline $\begin{array}{l}\text { TLG } \\
\text { Median } \\
\text { Interquartile range }\end{array}$ & $\begin{array}{l}87.8 \\
41.9-183.3\end{array}$ & $\begin{array}{l}- \\
- \\
-\end{array}$ & \\
\hline
\end{tabular}

Notes: ${ }^{2}$ Two-sample $t$-tests were used to compare age and plasma glucose differences between groups; ${ }^{\mathrm{b}} \mathrm{Chi}$-square tests were used to compare sex differences; For all tests, $p<0.05$ was considered statistically significant; ${ }^{~} M a n n-W h i t n e y ~ U$-tests were used to compare the injected dose/weight differences between control subjects and patients.

Abbreviations: aaIPI/IPI, age-adjusted international prognostic index/international prognostic index; EBVDNA, Epstein-Barr virus deoxyribonucleic acid; ECOG PS, Eastern Cooperative Oncology Group performance status; LDH, lactate dehydrogenase; $\beta 2$-MG, $\beta 2$-microglobulin; MTV, metabolic tumor volume; PINKE, prognostic index of natural killer lymphoma and EBV-DNA; SUVmax, maximum standard uptake value; TLG, total lesion glycolysis; ULN, upper limit of normal.

radiotherapy as first-line treatment. They were followed up until March 1, 2019. During the median follow-up of 24.53 (range, 0.53-103.7) months, 15 patients died, 3 patients survived with disease, and 42 patients survived without disease. The median PFS and OS were 21.67 months (95\% confidence interval [CI] 22.65-36.04) and 24.53 months (95\% CI 25.52-38.63), respectively. The patients were divided into two subgroups: a survival 


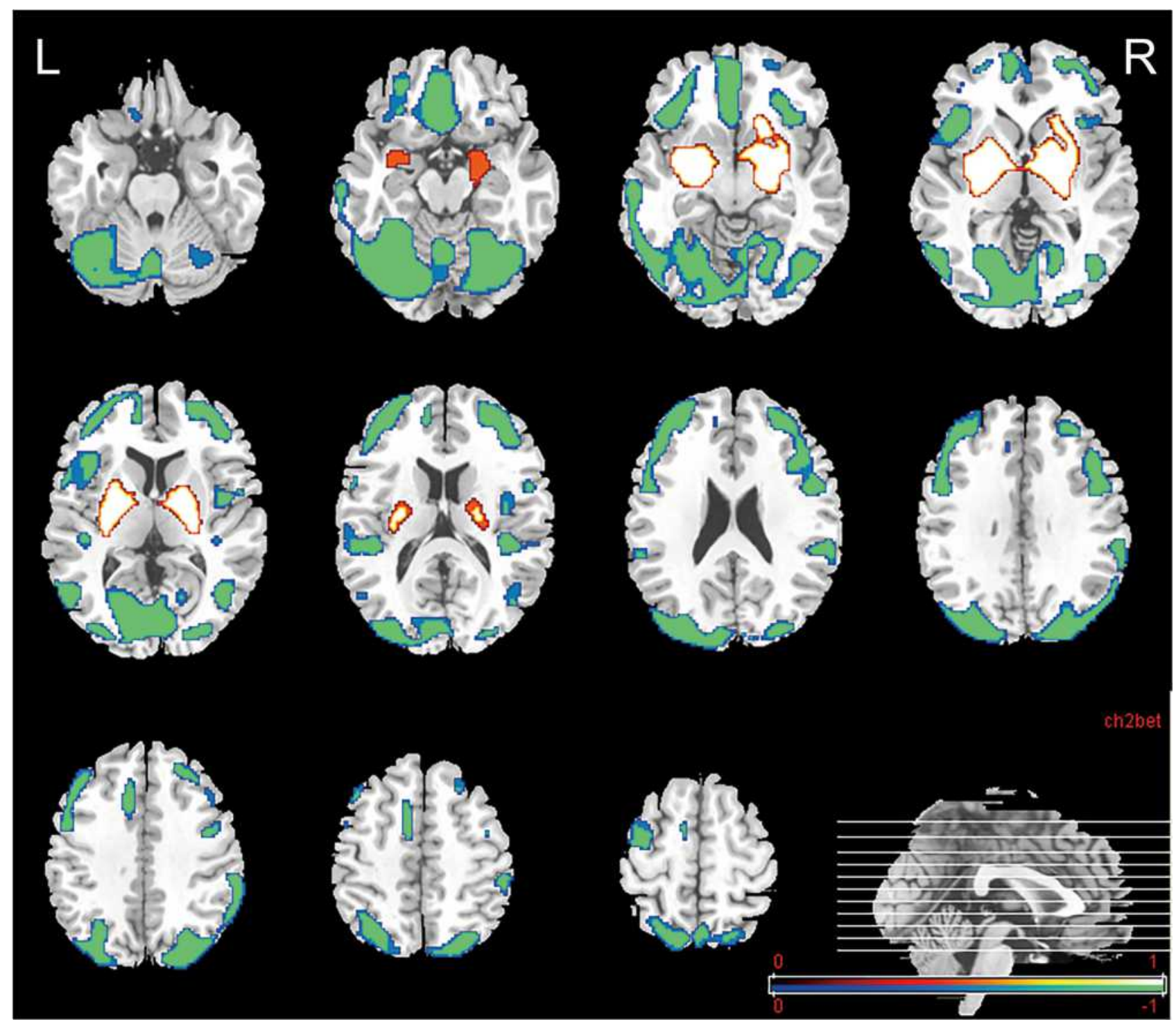

Figure 2 Abnormal glucose uptake in ENKTL patients compared with the CG. PET findings were superimposed on an MRI template to facilitate accurate identification of the affected structures. The increased regions are shown in red-to-white color and include both bilateral lentiform nuclei with slight extension to the adjacent claustrum and parahippocampal gyrus, especially in the putamen, amygdala, and hippocampus. The decreased regions are displayed with blue-to-green color and include the bilateral frontal cortex, parietal cortex, occipital cortex, temporal cortex, and cerebellum $(p<0.00 \mathrm{I})$. Color bar indicates t-values.

Abbreviations: L, left; $R$, right.

group (45 patients, average age of 50.9 years, 14 females and 31 males) and a death group (15 patients, average age of 46 years, 4 females and 11 males).

\section{Comparison of Brain Glucose Metabolism}

The SPM results showed abnormal glucose uptake in stage I/ II ENKTL patients without any lesion identified on brain structural imaging. Compared to the $\mathrm{CG}$, the regional cerebral glucose metabolism of pretreatment patients was decreased in the bilateral frontal, parietal, occipital, temporal cortex, and cerebellum, with especially large difference in the prefrontal cortex (PFC), orbitofrontal cortex (OFC), partial parietal and occipital cortex, cingulate gyrus, and cerebellum (Figure 2 and Table 2). An increase in metabolism was also observed in a relatively solitary focal area involving the bilateral basal ganglia and hippocampus (Figure 2 and Table 3). The remaining areas were largely spared from changes, especially the sensorimotor cortex.

Compared with the survival group, regional cerebral glucose metabolism was higher in the bilateral amygdala, bilateral putamen, left thalamus, and left parahippocampal gyrus (especially left uncus) in the death group (Figure 3 and Table 4). However, there were no areas with significantly lower metabolism (Figure 3). 
Table 2 Decreased Glucose Metabolism Regions in Patients Compared with Controls

\begin{tabular}{|c|c|c|c|c|c|}
\hline \multirow[t]{2}{*}{ Brain Regions } & \multicolumn{2}{|c|}{ Peak Level } & \multicolumn{3}{|c|}{$\begin{array}{l}\text { Peak Coordinates } \\
\text { x, y, z }(\mathrm{mm})\end{array}$} \\
\hline & $\mathbf{p}$ & $\mathbf{T}$ & $\mathbf{x}$ & $\mathbf{y}$ & $\mathbf{z}$ \\
\hline Bil. superior frontal gyrus & $<0.001$ & 3.98 & 8 & II & 48 \\
\hline Bil. middle frontal gyrus & $<0.001$ & 4.24 & -24 & 9 & 59 \\
\hline Bil. inferior frontal gyrus & $<0.001$ & 5.10 & -42 & 39 & 7 \\
\hline Bil. orbital gyrus & $<0.001$ & 3.16 & 8 & 43 & 18 \\
\hline Bil. parietal lobe, precuneus & $<0.001$ & 4.77 & -4 & -63 & 60 \\
\hline Bil. occipital lobe, cuneus & $<0.001$ & 6.77 & 30 & -74 & -11 \\
\hline Rt. limbic lobe (cingulate gyrus) & $<0.001$ & 3.88 & 8 & 2 & 46 \\
\hline Bil. temporal lobe, fusiform gyrus & $<0.001$ & 6.99 & 44 & -61 & -12 \\
\hline Bil. insula & $<0.001$ & 4.00 & 55 & -34 & 18 \\
\hline Bil. cerebellum & $<0.001$ & 6.58 & 34 & -57 & -14 \\
\hline
\end{tabular}

Table 3 Increased Glucose Metabolism Regions in Patients Compared with Controls

\begin{tabular}{|l|c|c|c|c|c|}
\hline \multirow{2}{*}{ Brain Regions } & \multicolumn{2}{|c|}{ Peak Level } & \multicolumn{3}{|c|}{$\begin{array}{c}\text { Peak } \\
\text { Coordinates } \\
\mathbf{x , y}, \mathbf{z}(\mathbf{m m})\end{array}$} \\
\cline { 2 - 6 } & $\mathbf{P}$ & $\mathbf{T}$ & $\mathbf{x}$ & $\mathbf{y}$ & $\mathbf{z}$ \\
\hline $\begin{array}{l}\text { Bil. lentiform nucleus, globus } \\
\text { pallidus }\end{array}$ & $<0.001$ & 7.21 & -16 & -4 & 2 \\
\hline Bil. lentiform nucleus, putamen & $<0.001$ & 4.26 & -18 & 19 & -8 \\
\hline Bil. claustrum & $<0.001$ & 3.74 & -32 & 6 & -4 \\
\hline Bil. amygdala & $<0.001$ & 3.64 & -20 & -5 & -13 \\
\hline Bil. hippocampus & $<0.001$ & 4.09 & -26 & -12 & -8 \\
\hline
\end{tabular}

\section{Correlations Between Brain Glucose} Metabolism Metrics and Other Parameters

The multiple linear regression analysis results are shown in Table 5. Only $B$ symptoms $\left(\mathrm{R}=0.642, \mathrm{R}^{2}=0.412\right.$, $\mathrm{P}=0.002$ ) may affect metabolism in the RBGT. No other correlation between regional cerebral glucose metabolism and clinical characteristics was found.

\section{Comparison of the PET/CT Metabolic Parameters}

The baseline PET/CT metabolic parameters of all patients were as follows: median SUVmax 12.2 (range, 8.5-16.1), median MTV $12.2 \mathrm{~cm}^{3}$ (range, 7.1-20.9), median of TLG
87.8 (range, 41.9-183.3). The Median SUVmaxRBGT/ SUVmaxLC (left cerebellum) was 1.43 (range, 1.38-1.51), SUVmaxLBGT/SUVmaxRC was 1.43 (range, 1.35-1.48), SUVmaxRU (regions around right uncus)/SUVmaxLC was 0.721 (range, 0.688-0.766), SUVmaxLU/ SUVmaxRC was 0.702 (range, 0.676-0.772). Optimal cutoff values of MTV, TLG, and SUVmaxLBGT/ SUVmaxRC for OS were $9.21 \mathrm{~cm}^{3}$ (area under the curve [AUC] $=0.775 ; \quad$ sensitivity $100 \%$; specificity $44.4 \%$; $\mathrm{P}=0.002), 160.46$ (AUC $=0.788$; sensitivity $66.7 \%$; specificity $82.2 \% ; \mathrm{P}=0.001$ ), and 1.52 ( $\mathrm{AUC}=0.650$; sensitivity $53.3 \%$; specificity $80.0 \%$; $\mathrm{P}=0.043$ ) in 60 patients with ENKTL, respectively. The AUCs for the other metabolic parameters were not significant for OS.

The population was dichotomized with the cut-off values/medians of PET/CT metabolic parameters. KaplanMeier curves are displayed in Figure 4. The median PFS (15.53 vs 33.22 months, $\mathrm{P}=0.003 ; 11.53$ vs 23.88 months, $\mathrm{P}=0.000 ; 14.20$ vs 22.30 months, $\mathrm{P}=0.017$ ) and the median OS (18.30 vs 36.73 months, $\mathrm{P}=0.002 ; 16.32$ vs 27.50 months, $\mathrm{P}=0.000 ; 18.03$ vs 25.93 months; $\mathrm{P}=0.011$ ) were significantly different in patients with high and low MTV, TLG, SUVmaxLBGT/SUVmaxRC, respectively. There was also a significant difference in patients with high and low SUVmaxRBGT/SUVmaxLC with regard to the median OS (22.82 vs 30.28 months, $\mathrm{P}=0.029)$. The SUVmax and metabolism of LU and RU had no predictive value for PFS or OS.

\section{Comparison of Clinical and PET/CT Parameters}

Patient characteristics stratified according to cut-off values/medians of PET/CT parameters are presented in Table 6. According to $X^{2}$ or Fisher exact tests, patients with high SUVmax had more B symptoms, higher lactate dehydrogenase (LDH) levels, and IPI scores $(\mathrm{P}=0.008$, 0.021 and 0.007 , respectively). High MTV was associated with advanced stage and Eastern Cooperative Oncology Group (ECOG) scores ( $\mathrm{P}=0.010$ and 0.012 , respectively). High TLG was correlated with more B symptoms; higher $\mathrm{LDH}$ and $\beta 2$-microglobulin ( $\beta 2-\mathrm{MG})$ levels; and high ECOG, age-adjusted IPI (aaIPI)/IPI and PINKE scores ( $\mathrm{P}=0.029,0.004,0.046,0.000,0.047$, and 0.010, respectively). Glucose metabolism of the RBGT and LBGT was noticeably higher in patients with more $\mathrm{B}$ symptoms, higher ECOG scores $(\mathrm{P}=0.008,0.015$, and 0.014, 0.013, respectively). Meanwhile, high LBGT metabolism was 


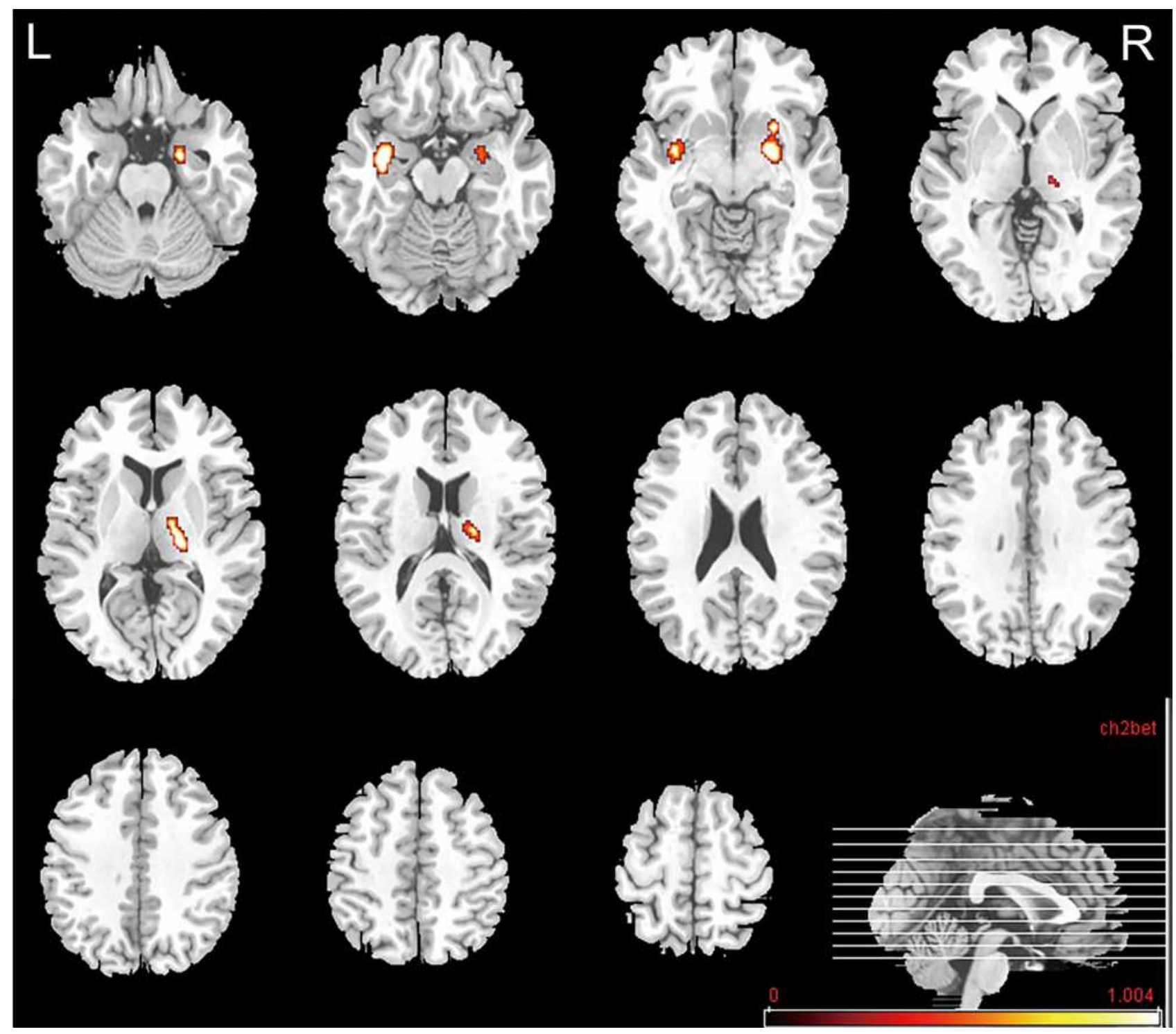

Figure 3 Abnormal glucose uptake in the death group compared with the survival group. The PET findings were superimposed on an MRI template to facilitate accurate identification of the affected structures. The cerebral glucose metabolism displayed with red-to-white color was significantly higher in the bilateral amygdala, bilateral putamen, left thalamus, and left uncus $(\mathrm{p}<0.00 \mathrm{I})$. No significantly lower metabolism was found. Color bar indicates $t$-values.

Abbreviations: $L$, left; $R$, right.

associated with high PINKE scores $(\mathrm{P}=0.022)$. LU and RU glucose metabolism were not correlated with any clinical parameters.

The univariate analyses results for PFS and OS using the clinical variables and PET parameters are shown in Table 7. The variables significantly associated with both PFS and OS were TLG, B symptoms, LDH, ECOG1-2, aaIPI/IPI1-2, SUVmaxLBGT/SUVmaxRC. We found that SUVmaxRBGT/SUVmaxLC was predictive of shorter OS. The difference in laterality may be related to the dominant hand. MTV was predictive of shorter PFS. Parameters significantly associated with PFS and OS were entered into a multivariate Cox proportional hazards model. The results showed that only ECOG1-2 was an independent predictor of both shorter PFS and OS. PINKE 1-2 was an independent predictor of OS in stage I/II ENKTL patients.

\section{Discussion}

Our study revealed abnormal regional cerebral glucose metabolism in pretreatment stage I/II ENKTL patients with limited lesions and low TLG, which might not be due to glucose competition as previously proposed. ${ }^{6}$ Only B symptoms were associated with increased metabolism in the RBGT. We also found that stage I/II ENKTL patients with different prognoses had variable brain glucose metabolism patterns before treatment. Higher pretreatment 
Table 4 Increased Glucose Metabolism Regions in Decreased Patients Compared to Surviving Patients

\begin{tabular}{|l|c|c|c|c|c|}
\hline \multirow{2}{*}{ Brain Regions } & \multicolumn{3}{|c|}{ Peak Level } & \multicolumn{2}{c|}{$\begin{array}{c}\text { Peak Coordinates } \\
\mathbf{x}, \mathbf{y}, \mathbf{z}(\mathbf{m m})\end{array}$} \\
\cline { 2 - 6 } & $\mathbf{P}$ & $\mathbf{T}$ & $\mathbf{x}$ & $\mathbf{y}$ & $\mathbf{z}$ \\
\hline Bil. amygdala & $<0.00 \mathrm{I}$ & 3.95 & 34 & -8 & -13 \\
\hline Bil. putamen & $<0.00 \mathrm{I}$ & 3.33 & -26 & $\mathrm{II}$ & -9 \\
\hline Lt. parahippocampal gyrus & $<0.00 \mathrm{I}$ & 3.50 & -22 & -7 & -22 \\
\hline Lt. limbic lobe, uncus & $<0.00 \mathrm{I}$ & 3.50 & -22 & -3 & -28 \\
\hline Lt. thalamus & $<0.00 \mathrm{I}$ & 3.84 & -16 & -13 & 12 \\
\hline
\end{tabular}

glucose metabolism in the BGT and some clinical parameters may be useful for restratifying and reclassifying stage I/II ENKTL patients.

Based on the observed abnormal regional cerebral glucose metabolism, we propose several mechanisms to this phenomenon. First, the most plausible mechanism is the auto-immunogenic reaction known as paraneoplastic neurologic syndromes (PNS). Up to $10 \%$ of patients with lymphoma may develop PNS. ${ }^{15}$ Similar abnormal cerebral metabolism including focal sparing of the sensorimotor cortex was observed in patients with systemic lupus erythematosus ${ }^{16}$ and PNS. ${ }^{17}$ The basal ganglia, amygdala, and thalamus are major visceral to brain signal transduction pathways and part of the neuroendocrine-immune system, which helps the brain control tumor growth in peripheral tissues. ${ }^{18,19}$ Increased metabolism might indicate their activation. Hypometabolism of the bilateral parietal, occipital, and medial frontal-associated cortices might be associated with the impairments of spatial orientation function and attention induced by PNS in our patients. ${ }^{19,20}$

Depression and post-traumatic stress disorder, which are prevalent in cancer patients, ${ }^{21,22}$ might be another mechanism. The hippocampus, amygdala, and putamen are part of the ventral "emotion" circuit ${ }^{22}$ that can be activated by negative emotions. ${ }^{2,5,18,23-25}$ Decreased cerebral blood flow, decreased metabolism, ${ }^{1,2}$ and reduction of gray matter volume ${ }^{26,27}$ in the PFC, OFC, and anterior cingulate cortex were also observed in depressive patients. Notably, these changes were proportional to depression severity ${ }^{26}$ and could be partly recovered with attenuation of psychiatric symptoms ${ }^{1,17,28}$ due to an effective therapeutic response. ${ }^{2,29}$

Table 5 Multiple Linear Regression Analysis Between Brain Glucose Metabolism Metrics and PET/CT Parameters

\begin{tabular}{|c|c|c|c|c|c|c|c|c|}
\hline & \multicolumn{2}{|c|}{$\begin{array}{c}\text { SUVmax RBGT/SUVmax } \\
\text { LC }\end{array}$} & \multicolumn{2}{|c|}{$\begin{array}{c}\text { SUVmax LBGT/SUVmax } \\
\text { RC }\end{array}$} & \multicolumn{2}{|c|}{$\begin{array}{c}\text { SUVmax RU/SUVmax } \\
\text { LC }\end{array}$} & \multicolumn{2}{|c|}{$\begin{array}{c}\text { SUVmax LU/SUVmax } \\
\text { RC }\end{array}$} \\
\hline & $R=0.642$ & $R^{2}=0.412$ & $R=0.530$ & $\mathrm{R}^{2}=0.28 \mathrm{I}$ & $R=0.4 I I$ & $R^{2}=0.169$ & $R=0.323$ & $R^{2}=0.105$ \\
\hline & Coefficient & p-value & Coefficient & p-value & Coefficient & p-value & Coefficient & p-value \\
\hline TLG lesion & 0.000 & 0.182 & 0.000 & 0.456 & 0.000 & 0.444 & 0.000 & 0.527 \\
\hline Sex & -0.130 & 0.060 & -0.043 & 0.557 & -0.026 & 0.581 & 0.050 & 0.408 \\
\hline Age & -0.318 & 0.086 & -0.252 & 0.208 & 0.044 & 0.734 & -0.017 & 0.918 \\
\hline Stage (I/II) & -0.318 & 0.490 & 0.027 & 0.695 & 0.018 & 0.693 & 0.019 & 0.745 \\
\hline B symptoms & 0.232 & 0.002 & 0.129 & 0.094 & 0.065 & 0.192 & -0.016 & 0.801 \\
\hline ECOG PS & 0.077 & 0.077 & 0.079 & 0.355 & -0.004 & 0.940 & -0.019 & 0.780 \\
\hline LDH & -0.222 & 0.226 & -0.068 & 0.731 & -0.005 & 0.969 & 0.012 & 0.942 \\
\hline EBVDNA & -0.088 & 0.477 & 0.017 & 0.899 & 0.088 & 0.313 & 0.065 & 0.558 \\
\hline$\beta 2-M G$ & -0.039 & 0.553 & -0.070 & 0.327 & -0.018 & 0.692 & -0.027 & 0.646 \\
\hline aalPI/IPI & 0.222 & 0.230 & 0.136 & 0.498 & 0.015 & 0.909 & 0.055 & 0.738 \\
\hline PINKE & -0.157 & 0.230 & -0.113 & 0.426 & -0.178 & 0.056 & -0.146 & 0.209 \\
\hline
\end{tabular}

Abbreviations: aalPI/IPI, age-adjusted international prognostic index/international prognostic index; EBVDNA, Epstein-Barr virus deoxyribonucleic acid; ECOG PS, Eastern Cooperative Oncology Group performance status; LBGT, left basal ganglia and thalamus; LC, left cerebellum; LDH, lactate dehydrogenase; LU, regions around left uncus; $\beta 2-M G, \beta 2$-microglobulin; MTV, metabolic tumor volume; PINKE, prognostic index of natural killer lymphoma and EBV-DNA; RBGT, right basal ganglia and thalamus; RC, right cerebellum; RU, regions around right uncus; SUVmax, maximum standard uptake value; TLG, total lesion glycolysis. 

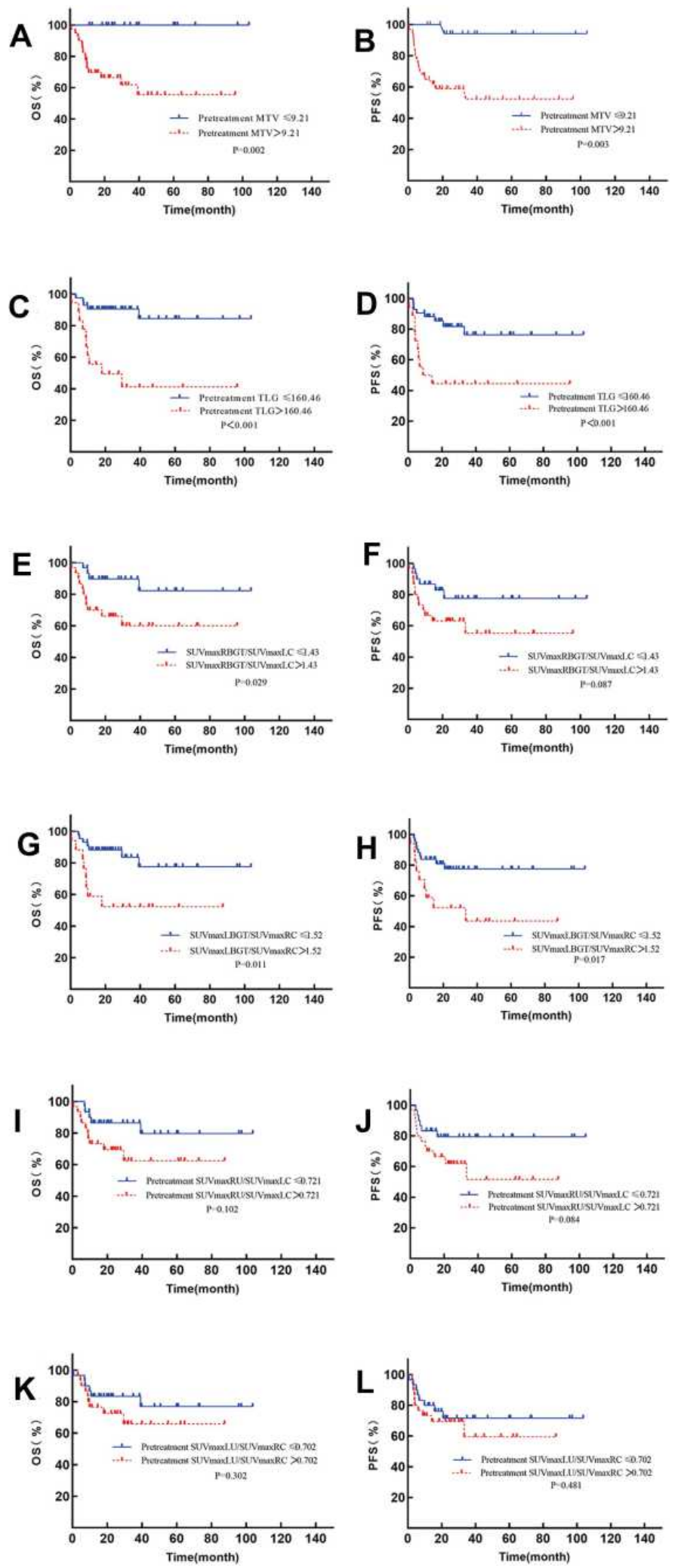

Figure 4 Kaplan-Meier estimates of PFS and OS. Survival outcomes estimated by using pretreatment MTV: OS in ENKTL (A) and PFS in ENKTL (B); survival outcomes according to pretreatment TLG: OS (C) and PFS (D); survival outcomes according to SUVmaxRBGT/SUVmaxLC: OS (E) and PFS (F); survival outcomes according to SUVmaxLBGT/SUVmaxRC: OS (G) and PFS (H); survival outcomes according to SUVmaxRU/SUVmaxLC: OS (I) and PFS (J); survival outcomes according to SUVmaxLU/ SUVmaxRC: OS (K) and PFS (L).

Abbreviations: LBGT, left basal ganglia and thalamus; RBGT, right basal ganglia and thalamus; LC, left cerebellum; RC, right cerebellum; LU, left regions around right uncus; $\mathrm{RU}$, right regions around right uncus. 
Table 6 Relationships Between Clinical Characteristics and PET/CT Parameters

\begin{tabular}{|c|c|c|c|c|c|c|c|c|c|c|c|c|c|c|c|}
\hline & \multicolumn{3}{|c|}{$\begin{array}{l}\text { SUVmax } \\
\text { Lesion }\end{array}$} & & \multicolumn{3}{|c|}{ MTV Lesion } & & \multicolumn{2}{|c|}{ TLG Lesion } & & \multicolumn{3}{|c|}{$\begin{array}{l}\text { SUVmax } \\
\text { RBGT/SUVmax } \\
\text { LC }\end{array}$} & \\
\hline & $\leq \mathbf{I} 2.23$ & \multicolumn{2}{|c|}{$>12.23$} & & $\leq 9.21$ & \multicolumn{2}{|c|}{$>9.21$} & & $\leq 160.46$ & $>160.46$ & & $\leq \mathrm{I} .43$ & \multicolumn{2}{|c|}{$>1.43$} & \\
\hline & $n=30$ & \multicolumn{2}{|c|}{$n=30$} & $p$ & $\mathbf{n}=\mathbf{2 0}$ & \multicolumn{2}{|c|}{$n=40$} & $p$ & $n=42$ & $n=18$ & $p$ & $n=30$ & \multicolumn{2}{|c|}{$n=30$} & $p$ \\
\hline Gender (M/F) & $20 / 10$ & \multicolumn{2}{|l|}{$22 / 8$} & 0.573 & $13 / 7$ & \multicolumn{2}{|c|}{$29 / 11$} & 0.550 & $28 / 14$ & $14 / 4$ & 0.389 & $22 / 8$ & \multicolumn{2}{|c|}{$20 / 10$} & 0.573 \\
\hline Age $(\leq 60 />60)$ & $26 / 4$ & \multicolumn{2}{|l|}{$21 / 9$} & 0.209 & $15 / 5$ & \multicolumn{2}{|c|}{$32 / 8$} & 0.658 & $34 / 8$ & $13 / 5$ & 0.452 & $20 / 10$ & \multicolumn{2}{|l|}{$27 / 3$} & 0.057 \\
\hline Stage (I/II) & $15 / 15$ & \multicolumn{2}{|c|}{$13 / 17$} & 0.605 & $14 / 6$ & $4 / 2$ & & 0.01 & $22 / 20$ & $6 / 12$ & 0.175 & $15 / 15$ & $13 / 1$ & & 0.605 \\
\hline B symptoms (No/Yes) & $23 / 7$ & $13 / 17$ & & 0.008 & $15 / 5$ & 21 & & 0.09 & $29 / 13$ & $7 / 11$ & 0.029 & $23 / 7$ & $13 / 1$ & & 0.008 \\
\hline ECOG PS (0/I-2) & $25 / 5$ & $20 / I C$ & & 0.136 & $19 / 1$ & 26 & & 0.01 & $37 / 5$ & $8 / 10$ & 0.000 & $27 / 3$ & $18 / 1$ & & 0.015 \\
\hline LDH $(\leq \mathrm{ULN} />\mathrm{ULN})$ & $28 / 2$ & $20 / I C$ & & 0.021 & $18 / 2$ & 30 & & 0.30 & $38 / 4$ & $10 / 8$ & 0.004 & $27 / 3$ & $21 / 9$ & & 0.104 \\
\hline EBVDNA $(\leq U L N />U L N)$ & $22 / 8$ & $25 / 5$ & & 0.347 & $18 / 2$ & 29 & & 0.18 & $35 / 7$ & $12 / 6$ & 0.151 & $23 / 7$ & $24 / 6$ & & 0.754 \\
\hline$\beta 2-M G(\leq U L N />U L N)$ & $19 / 11$ & $16 / 1$ & & 0.432 & $14 / 6$ & $21 /$ & & 0.19 & $28 / 14$ & $7 / 11$ & 0.046 & $20 / 10$ & $15 / 1$ & & 0.190 \\
\hline aalPI/IPI (0/I-2) & $24 / 6$ & $14 / 16$ & & 0.007 & $13 / 7$ & 25 & & 0.85 & $30 / 12$ & $8 / 10$ & 0.047 & $19 / 11$ & $19 / 1$ & & 1.000 \\
\hline PINKE (0/I-2) & $19 / 11$ & $16 / 1$ & & 0.432 & $14 / 6$ & $21 /$ & & 0.19 & $29 / 13$ & $6 / 12$ & 0.010 & $14 / 16$ & $21 / 9$ & & 0.067 \\
\hline Characteristics & SUVma & $x$ LBC & ST/S & UVma & RC & & & Vmax & x RU/SUVma & $\times \mathbf{L C}$ & SUVm & $x$ LU/SI & $V \max$ & RC & \\
\hline & $\leq \mathbf{I . 5 2}$ & & $>1.5$ & & & & & & $>0.721$ & & $\leq 0.702$ & & 702 & & \\
\hline & $n=43$ & & $n=1$ & & $p$ & & $\mathrm{n}=$ & & $n=30$ & $p$ & $n=30$ & $\mathbf{n}=$ & & $p$ & \\
\hline $\operatorname{Sex}(M / F)$ & $30 / 13$ & & $12 / 5$ & & 0. & 50 & 21 & & $21 / 9$ & 1.000 & $21 / 9$ & 21 & & 1.0 & \\
\hline Age $(\leq 60 />60)$ & $32 / 11$ & & $15 / 2$ & & 0.7 & 42 & 20 & & $27 / 3$ & 0.057 & $20 / 10$ & 27 & & 0.0 & \\
\hline Stage $(I / I I)$ & $21 / 22$ & & $7 / 10$ & & 0.5 & 92 & 15 & & $13 / 17$ & 0.605 & $15 / 15$ & 13 & & 0.6 & \\
\hline B symptoms (No/Yes) & $30 / 13$ & & $6 / 11$ & & $0 .($ & 14 & 22 & & $14 / 16$ & 0.035 & $19 / 11$ & 17 & & 0.5 & \\
\hline ECOG PS (0/I-2) & $36 / 7$ & & $9 / 8$ & & 0. & 13 & 26 & & $19 / 11$ & 0.072 & $24 / 6$ & 21 & & 0.3 & \\
\hline LDH $(\leq \mathrm{ULN} />\mathrm{ULN})$ & $36 / 7$ & & $12 / 5$ & & 0.7 & 52 & 18 & & $20 / 10$ & 0.592 & $25 / 5$ & 23 & & 0.5 & \\
\hline EBVDNA $(\leq U L N />U L N)$ & $31 / 12$ & & $16 / 1$ & & 0. & & 22 & & $25 / 5$ & 0.347 & $24 / 6$ & 23 & & 0.7 & \\
\hline$\beta 2-M G(\leq U L N />U L N)$ & $26 / 17$ & & $9 / 8$ & & 0.5 & 94 & $|7|$ & & $18 / 12$ & 0.793 & $15 / 15$ & 20 & & 0.1 & \\
\hline aalPI/IPI (0/I-2) & $28 / 15$ & & $10 / 7$ & & 0. & 49 & 18 & & $20 / 10$ & 0.592 & $17 / 13$ & 21 & & 0.2 & \\
\hline PINKE (0/I-2) & $21 / 22$ & & $14 / 3$ & & & 22 & 13 & & $22 / 8$ & 0.018 & $15 / 15$ & 20 & & 0.1 & \\
\hline
\end{tabular}

Abbreviations: aalPI/IPI, age-adjusted international prognostic index/international prognostic index; EBVDNA, Epstein-Barr virus deoxyribonucleic acid; ECOG PS, Eastern Cooperative Oncology Group performance status; LC, left cerebellum; LDH, lactate dehydrogenase; $\beta 2$-MG, $\beta 2$-microglobulin; MTV, metabolic tumor volume; PINKE, prognostic index of natural killer lymphoma and EBV-DNA; RC, right cerebellum; SUVmax, maximum standard uptake value; TLG, total lesion glycolysis.

Finally, cognitive impairment could be another mechanism. It was reported associated with depression and chemobrain, ${ }^{30}$ and such cerebral metabolism could see in Alzheimer's disease too. ${ }^{1,17}$

Univariate analysis for PFS and OS revealed that MTV, TLG, SUVmaxLBGT/SUVmaxRC, SUVmaxRBGT/ SUVmaxLC, and multiple clinical factors were significantly associated with prognosis. In the subsequent multivariate analysis, only ECOG and PINKE scores were independent prognostic factors. Increased metabolism in the BGT might be associated with a worse outcome, but it did not surpass the predictive values of the ECOG and PINKE scores in our study. Although the exact mechanism remains unclear, it may indicate subclinical brain damage 


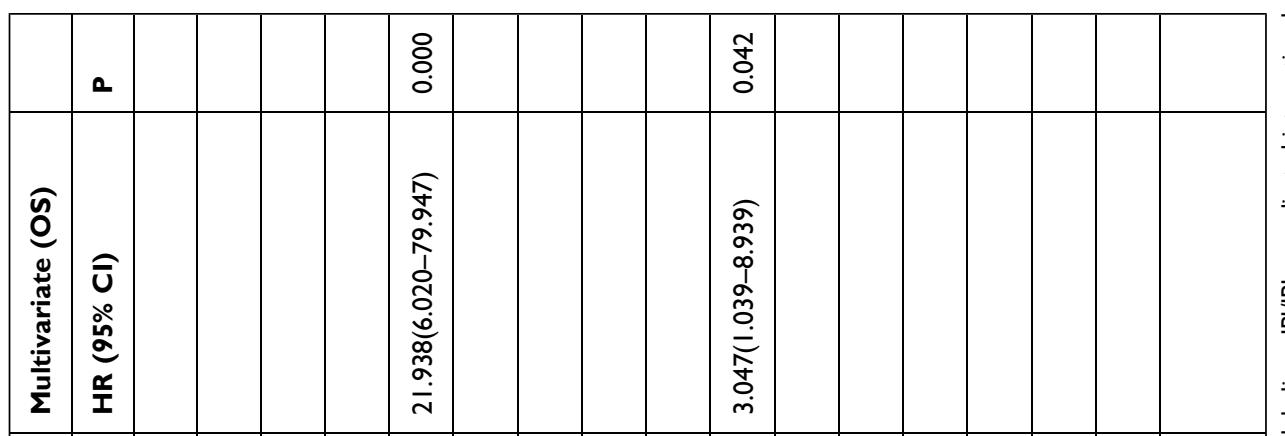

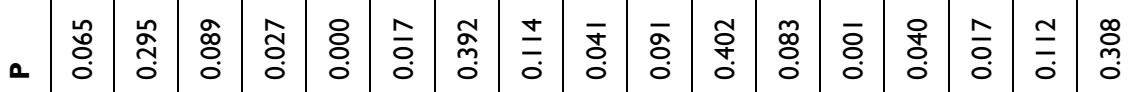

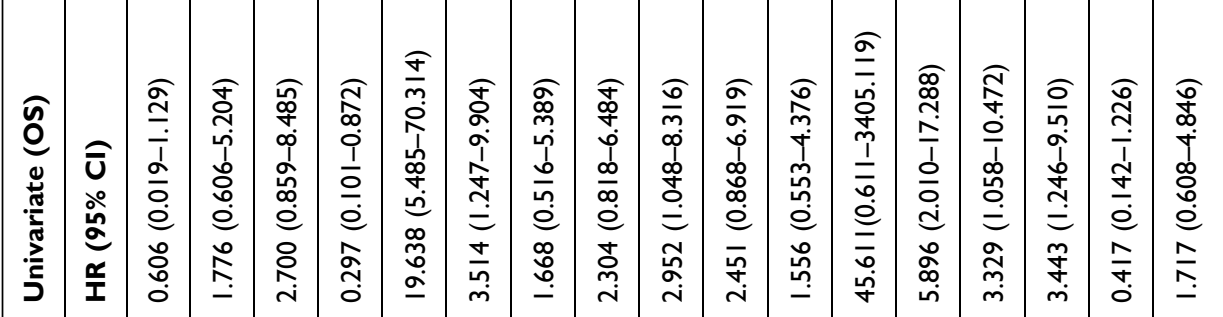

:

:

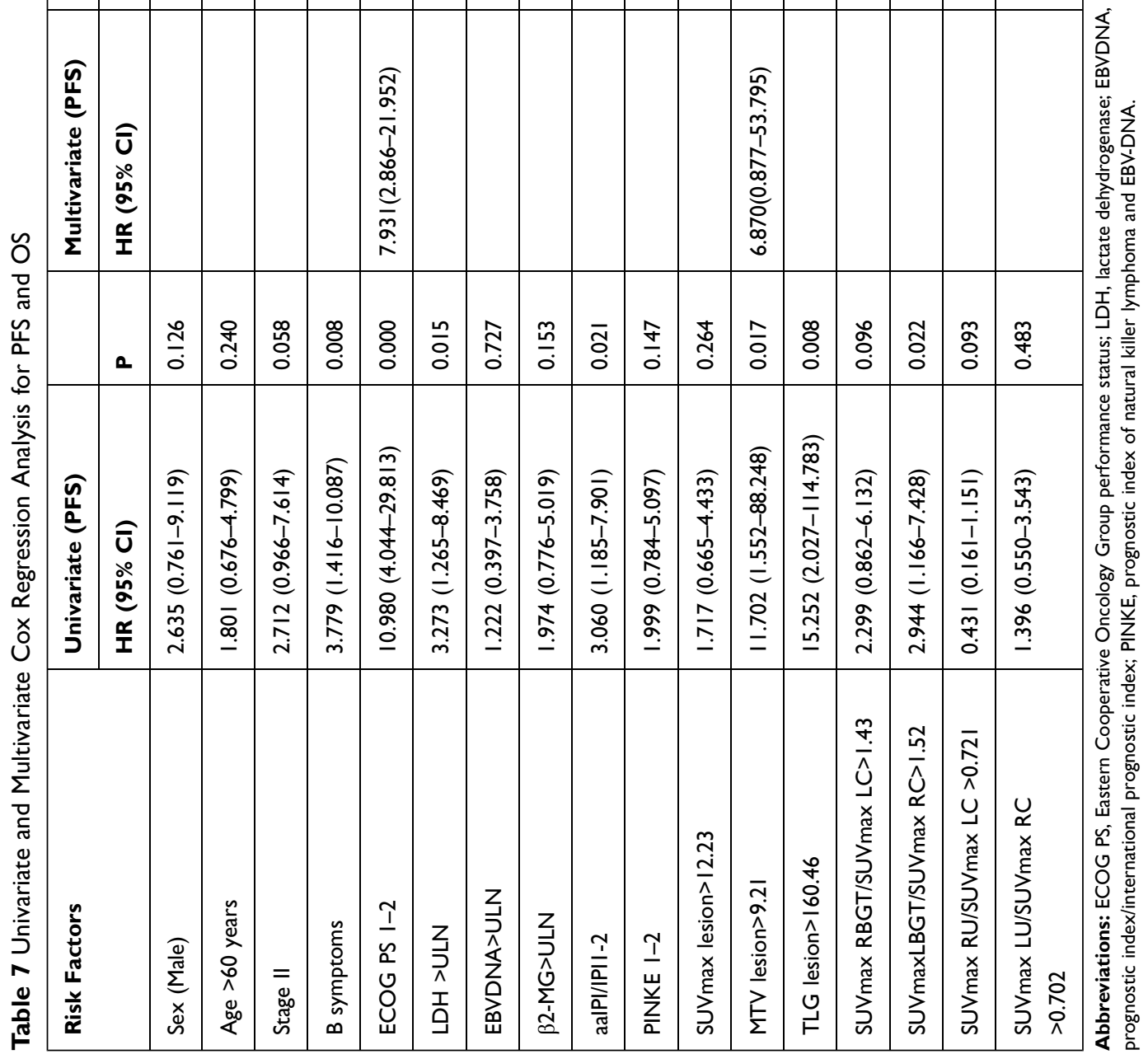


or functional alterations in stage I/II ENKTL patients. There is no recognized prognostic indicator or model for risk stratification of ENKTL patients, ${ }^{10,12,13}$ especially those who are stage I/II. Pretreatment cerebral glucose metabolism assessment is a routine, non-invasive method that may be a powerful, supplementary approach to restratify ENKTL patients. Moreover, it might facilitate the use of functional imaging as a supplementary diagnostic method for psychological evaluation of cancer patients in the future.

The results must be considered in the context of several limitations. The most serious is the retrospective design. In addition, it was difficult to control all the variables such as sleep hours. Larger and more rigorous prospective studies are needed to confirm our findings and optimize treatment regimens to improve the prognosis of stage I/II ENKTL patients.

\section{Conclusions}

The present study demonstrated that pretreatment stage I/ II ENKTL patients exhibited abnormal regional cerebral glucose metabolism. Increased metabolism in the BGT and high TLG and MTV of lesions were predictive factors of PFS and OS in stage I/II ENKTL patients; however, it did not surpass the predictive value of the ECOG and PINKE scores in this small cohort of patients. Future larger studies are required to further investigate the prognostic value of cerebral glucose metabolism in this patient population.

\section{Author Contributions}

All authors made a significant contribution to the work reported, whether that is in the conception, study design, execution, acquisition of data, analysis and interpretation, or in all these areas; took part in drafting, revising or critically reviewing the article; gave final approval of the version to be published; have agreed on the journal to which the article has been submitted; and agree to be accountable for all aspects of the work.

\section{Funding}

This study was supported by the Jiangsu Key Medical Talents Fund (ZDRCB20160003).

\section{Disclosure}

The authors report no conflicts of interest in this work.

\section{References}

1. Lin M, Huang G, Sun X, Ni J. The preliminary study of brain metabolic changes in lung cancer patients of different stages. Chin J Clin Med. 2009;16(4):516-518.

2. Tashiro M, Kubota K, Itoh M, et al. Regional cerebral glucose metabolism of patients with malignant diseases in different clinical phases. Med Sci Monit. 2001;7(2):226-232.

3. Ni J, Lin M, Liu J, Huang G. Regional brain metabolism changes in the body malignant tumor patients without brain metastasis. Chin $J$ Med Imaging Technol. 2010;26(11):2175-2178.

4. Machado AMC, Fagundes TC, Mafra A, Silva RG, Castro ACG, Mamede M. Effects on 18F-FDG PET/CT brain glucose metabolism in rectal cancer patients undergoing neoadjuvant chemotherapy. Clin Nucl Med. 2017;42:e484-e490. doi:10.1097/RLU.0000000000001862

5. Nonokuma M, Kuwabara Y, Takano K, Tamura K, Ishitsuka K, Yoshimitsu K. Evaluation of regional cerebral glucose metabolism in patients with malignant lymphoma of the body using statistical image analysis. Ann Nucl Med. 2014;28(10):950-960. doi:10.1007/ s12149-014-0890-1

6. Hanaoka K, Hosono M, Shimono T, et al. Decreased brain FDG uptake in patients with extensive non-Hodgkin's lymphoma lesions. Ann Nucl Med. 2010;24(10):707-711. doi:10.1007/s12149-010-0415-5

7. Chim CS, Ma SY, Au WY, et al. Primary nasal natural killer cell lymphoma: long-term treatment outcome and relationship with the international prognostic index. Blood. 2004;103(1):216-221. doi:10.1182/blood-2003-05-1401

8. Lee J, Suh C, Park YH, et al. Extranodal natural killer T-cell lymphoma, nasal-type: a prognostic model from a retrospective multicenter study. J Clin Oncol. 2006;24(4):612-618. doi:10.1200/ JCO.2005.04.1384

9. Yao N, Hou Q, Zhang S, et al. Prognostic nutritional index, another prognostic factor for extranodal natural killer/T cell lymphoma, nasal type. Front Oncol. 2020;10:877. doi:10.3389/fonc.2020.00877

10. Wang H, Shen G, Jiang C, Li L, Cui F, Tian R. Prognostic value of baseline, interim and end-of-treatment 18F-FDG PET/CT parameters in extranodal natural killer/T-cell lymphoma: a meta-analysis. PLoS One. 2018;13(3):e0194435. doi:10.1371/journal.pone.0194435

11. Guo R, Xu P, Xu H, Miao Y, Li B. The predictive value of pre-treatment 18 F-FDG $\mathrm{PET} / \mathrm{CT}$ on treatment outcome in early-stage extranodal natural killer/T-cell lymphoma. Leuk Lymphoma. 2020;61:2659-2664. doi:10.1080/ 10428194.2020.1783446

12. Qin C, Yang S, Sun X, Xia X, Li C, Lan X. 18F-FDG PET/CT for prognostic stratification of patients with extranodal natural killer/ T-cell lymphoma. Clin Nucl Med. 2019;44(3):201-208. doi:10.1097/RLU.0000000000002440

13. Huang JJ, Zhu YJ, Xia Y, et al. A novel prognostic model for extranodal natural killer/T-cell lymphoma. Med Oncol. 2012;29 (3):2183-2190. doi:10.1007/s12032-011-0030-x

14. Lister T. Report of a committee convened to discuss the evaluation and staging of patients with Hodgkin's disease: Cotswolds meeting. $J$ Clin Oncol. 1989;7:1630-1636. doi:10.1200/ JCO.1989.7.11.1630

15. Dalmau J, Rosenfeld MR. Paraneoplastic syndromes of the CNS. Lancet Neurol. 2008;7(4):327-340. doi:10.1016/S1474-4422(08) 70060-7

16. Mackay M, Tang CC, Volpe BT, et al. Brain metabolism and autoantibody titres predict functional impairment in systemic lupus erythematosus. Lupus Sci Med. 2015;2(1):e000074. doi:10.1136/ lupus-2014-000074

17. Clapp AJ, Hunt CH, Johnson GB, Peller PJ. Semiquantitative analysis of brain metabolism in patients with paraneoplastic neurologic syndromes. Clin Nucl Med. 2013;38(4):241-247. doi:10.1097/ RLU.0b013e3182815f28 
18. Chiaravalloti A, Pagani M, Cantonetti M, et al. Brain metabolic changes in Hodgkin disease patients following diagnosis and during the disease course: an (18)F-FDG PET/CT study. Oncol Lett. 2015;9 (2):685-690. doi:10.3892/ol.2014.2765

19. Zhang W, Ning N, Li X, et al. Changes of brain glucose metabolism in the pretreatment patients with non-small cell lung cancer: a retrospective PET/CT study. PLoS One. 2016;11(8):e0161325. doi:10.1371/journal.pone.0161325

20. D'Agata F, Costa T, Caroppo P, et al. Multivariate analysis of brain metabolism reveals chemotherapy effects on prefrontal cerebellar system when related to dorsal attention network. EJNMMI Res. 2013;3(1):22. doi:10.1186/2191-219X-3-22

21. Wedding U, Koch A, Rohrig B, et al. Depression and functional impairment independently contribute to decreased quality of life in cancer patients prior to chemotherapy. Acta Oncol. 2008;47 (1):56-62. doi:10.1080/02841860701460541

22. Mehnert A, Koch U. Prevalence of acute and post-traumatic stress disorder and comorbid mental disorders in breast cancer patients during primary cancer care: a prospective study. Psycho-oncol. 2007;16(3):181-188. doi:10.1002/pon.1057

23. Wefel JS, Lenzi R, Theriault R, Buzdar AU, Cruickshank S, Meyers CA. 'Chemobrain' in breast carcinoma? A prologue. Cancer. 2004;101(3):466-475. doi:10.1002/cncr.20393

24. Hsieh TC, Wu YC, Yen KY, Chen SW, Kao CH. Early changes in brain FDG metabolism during anticancer therapy in patients with pharyngeal cancer. $J$ Neuroimaging. 2014;24(3):266-272. doi:10.1111/jon.12006
25. Chiaravalloti A, Pagani M, Di Pietro B, et al. Is cerebral glucose metabolism affected by chemotherapy in patients with Hodgkin's lymphoma? Nucl Med Commun. 2013;34(1):57-63. doi:10.1097/ MNM.0b013e32835aa7de

26. Savitz J, Drevets WC. Bipolar and major depressive disorder: neuroimaging the developmental-degenerative divide. Neurosci Biobehav Rev. 2009;33(5):699-771.

27. Niida A, Niida R, Matsuda H, Inada T, Motomura M, Uechi A. Identification of atrophy of the subgenual anterior cingulate cortex, in particular the subcallosal area, as an effective auxiliary means of diagnosis for major depressive disorder. Int $J$ Gen Med. 2012;5:667-674. doi:10.2147/IJGM.S34093

28. Mosconi L, Tsui WH, Herholz K, et al. Multicenter standardized 18FFDG PET diagnosis of mild cognitive impairment, Alzheimer's disease, and other dementias. J Nucl Med. 2008;49(3):390-398. doi:10.2967/jnumed.107.045385

29. Temoshok LR. Psychological response and survival in breast cancer. Lancet. 2000;355(9201):404-405. doi:10.1016/S0140-6736(05) 74024-1

30. Kim CY, Hong CM, Kim DH, et al. Prognostic value of whole-body metabolic tumour volume and total lesion glycolysis measured on (1) (8)F-FDG PET/CT in patients with extranodal NK/T-cell lymphoma. Eur J Nucl Med Mol Imaging. 2013;40(9):1321-1329. doi:10.1007/ s00259-013-2443-6

\section{Publish your work in this journal}

OncoTargets and Therapy is an international, peer-reviewed, open access journal focusing on the pathological basis of all cancers, potential targets for therapy and treatment protocols employed to improve the management of cancer patients. The journal also focuses on the impact of management programs and new therapeutic agents and protocols on patient perspectives such as quality of life, adherence and satisfaction. The manuscript management system is completely online and includes a very quick and fair peer-review system, which is all easy to use. Visit http://www.dovepress.com/ testimonials.php to read real quotes from published authors. 